\title{
Ocular Pemphigus Vulgaris
}

\author{
M. Khamaily, J. Brahim Salem, H. Brarou, S. Dahi, \\ A. Omari, Y. Mouzari, F. El Asri, K. Reda, and A. Oubaaz
}

\begin{abstract}
Introduction: Pemphigus vulgaris is a rare intraepidermal autoimmune dermatosis, characterized by bullous cutaneous and mucosal involvement. Ocular involvement is relatively rare, of which blepharitis or conjunctivitis with hyperemia and secretions are the most described lesions

Methods: we report the case of a patient, who was hospitalized in dermatology for dermatological pemphigus with ocular involvement.

Results: the patient presented an erosive bilateral cutaneouspalpebral lesions with purulent secretions associated with conjunctival hyperemia

Conclusion: Ocular involvement in the common pemphigus remains an exception, the prognosis of which is often mild.
\end{abstract}

Index Terms - Pemphigus, blepharitis, ocular surface, autoimmune diseases.

\section{INTRODUCTION}

Pemphigus vulgaris is a rare intra-epidermal autoimmune dermatosis, characterized by bullous cutaneous and mucosal involvement [1]. Ocular involvement is relatively rare, of which blepharitis or conjunctivitis with hyperemia and secretions are the most described lesions [2], [3].

Apart from bacterial and especially viral (herpes) secondary infections, ocular damage during pemphigus is of good prognosis and which generally improves with the institution of systemic treatment of pemphigus. [4], [5].

\section{METHODS AND RESULTS}

We report the case of a 66-year-old patient, known to be diabetic for 15 years with oral anti-diabetics, who was hospitalized in dermatology for bullous cutaneous and mucous dermatosis (Fig. 1) histologically confirmed and in whom an ophthalmological examination was requested.

The AV with correction was 5/10 ODG.

With an erosive bilateral cutaneous-palpebral lesions with purulent secretions associated with conjunctival hyperemia. (Fig. 2).

Anterior segment: BUT decreased with diffuse KPS Anterior chamber: calm with nuclear cataract and posterior

Published on June 25, 2020

M. Khamaily, Mohammed V University of Rabat, Morocco.

(corresponding e-mail: mehdi.khamaily@gmail.com).

J. Brahim Salem, Mohammed V University of Rabat, Morocco.

H. Brarou, Mohammed V University of Rabat, Morocco.

S. Dahi, Mohammed V University of Rabat, Morocco.

A. Omari, Mohammed V University of Rabat, Morocco.

Y. Mouzari, Mohammed V University of Rabat, Morocco.

F. El Asri, Mohammed V University of Rabat, Morocco.

K. Reda, Mohammed V University of Rabat, Morocco.

A. Oubaaz, Mohammed V University of Rabat, Morocco. subcapsular.

Funds of the eye: without particularity.

Our treatment was based on eye wash with physiological saline with wetting agents and close clinical monitoring.

The patient progressed well under this treatment without serious eye damage.

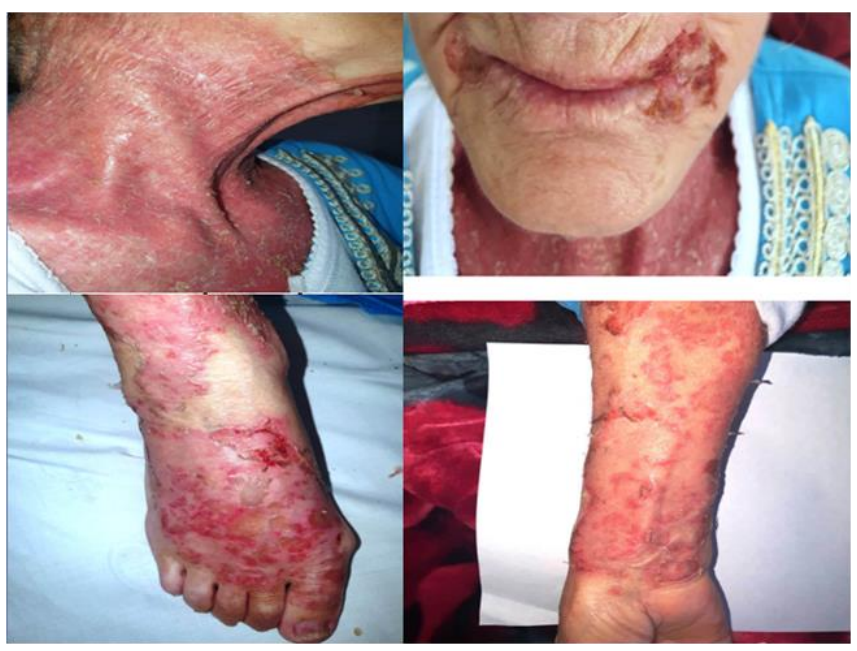

Fig. 1. Muco-cutaneous lesions: perioral, neck, upper and lower limb.

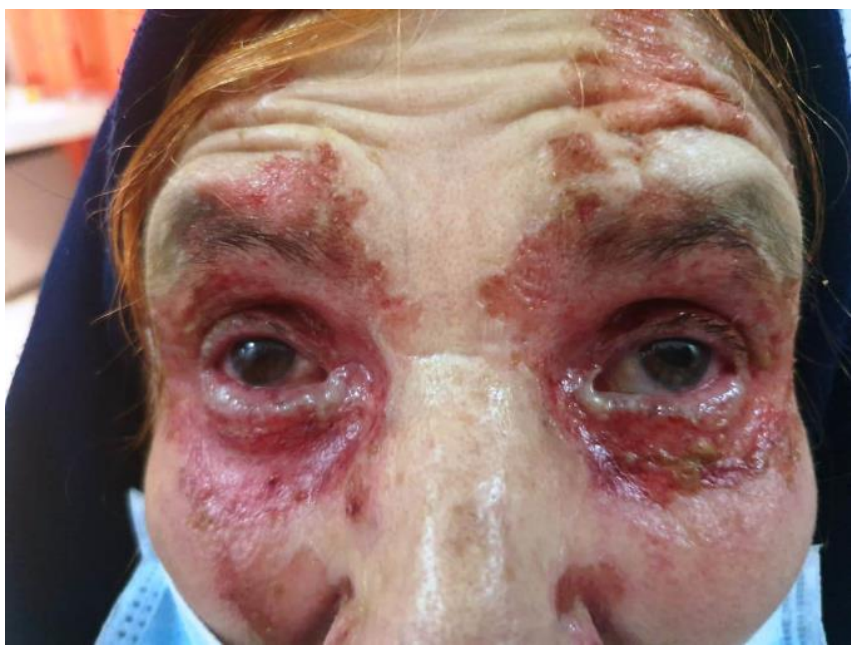

Fig. 2. Bilateral erosive palpebral lesion with conjunctival hyperemia

\section{CONCLUSION}

Ocular involvement in the common pemphigus remains an exception, the prognosis of which is often mild, and the treatment is systemic corticosteroids for dermatological involvement, as well as ocular hygiene measures and surface treatment. 


\section{REFERENCES}

[1] Eming R, et al. S2k guidelines for the treatment of pemphigus vulgaris/foliaceus and bullous pemphigoid. J Dtsch Dermatol Ges 2015; 13: 833-44.

[2] Elchahal $\mathrm{S}$ et al. Ocular manifestations of blis $\neg$ tering diseases. Immunol Allergy Clin N Am 2008; 28: 119-36.

[3] Brackley R, Pagani JM. Conjunctival erosions associated with pemphigus vulgaris. Optom Vis Sci 2011; 88: 1010-3.

[4] Palleschi G.M.et al. Ocular involvement in Pemphigus. Am J Ophtalmol 2007; 144:149-52.

[5] Agustin España, Pilar Iranzo, Josep Herrero-González, José M. Mascaro Jr., Ricardo Suárez. Ocular involvement in pemphigus vulgaris - a retrospective study of a large Spanish cohort. Deutsche Dermatologische Gesellschaft (DDG). Ltd. | JDDG | 1610-0379/2017. 